\title{
Industrielle Anwendungen von Goldkatalysatoren
}

\author{
Rosaria Ciriminna, Ermelinda Falletta, Cristina Della Pina, Joaquim \\ Henrique Teles* ${ }^{*}$ nd Mario Pagliaro*
}

\section{$\boldsymbol{E}_{r s t}$ kürzlich fanden Goldkatalysatoren den Weg in die ersten groß- technischen industriellen Anwendungen. Dieser Kurzaufsatz bietet eine kritische Analyse der Rahmenbedingungen und Hindernisse auf dem langen Weg von der Entdeckung der katalytischen Eigenschaften von Gold bis zur großtechnischen Anwendung von Goldkatalysato- ren. Diese Einblicke sollen Forschern aus Hochschule und Industrie, die heute an der Entwicklung von Goldkatalysatoren arbeiten, helfen, die Probleme von morgen zu bewältigen.}

\section{Einleitung}

Die Entdeckung des ersten goldhaltigen Katalysators, eines Katalysators für die oxidative Acetoxylierung von Ethylen zu Vinylacetat, liegt fast genau ein halbes Jahrhundert zurück. Das Patent, das diesen Katalysator beschreibt, wurde im Juni 1965 von der Deutschen Firma Knapsack eingereicht. ${ }^{[1]}$ Vier Jahrzehnte sind auch seit der Entdeckung von Bond und Sermon vergangen, dass die Aktivität von $\mathrm{Au} /$ $\mathrm{SiO}_{2}$ (erhalten durch schonendes Erhitzen von auf Siliciumoxid imprägniertem $\mathrm{HAuCl}_{4}$ ) für die Hydrierung von 1-Penten bei $373 \mathrm{~K}$ um den Faktor 7000 erhöht werden konnte, wenn der Goldgehalt von $1 \%$ auf $0.01 \%$ verringert wurde. ${ }^{[2]}$ Drei Jahrzehnte liegen auch zurück seit der fast gleichzeitigen Entdeckung durch Hutchings et al. ${ }^{[3]}$ und Haruta et al. ${ }^{[4]}$ dass auf Aktivkohle oder auf reduzierbaren oxidischen Trägern wie Titandioxid fixiertes, nanopartikuläres Gold ein außerordentlich effektiver Katalysator für die Hydrochlorierung von Acetylen und für die CO-Oxidation ist. Mit reduzierbaren oxidischen Trägern sind solche gemeint, die in der Lage sind, unter Bildung von Sauerstofffehlstellen Gittersauer-

[*] Dr. R. Ciriminna, Dr. M. Pagliaro

Istituto per lo Studio dei Materiali Nanostrutturati, CNR

via U. La Malfa 153, 90146 Palermo (Italien)

E-Mail:mario.pagliaro@cnr.it

Dr. E. Falletta, Prof. C. Della Pina

Dipartimento di Chimica, Università degli Studi di Milano via Venezian 21, Mailand (Italien)

Dr. J. H. Teles

BASF SE, Process Research and Chemical Engineering

67056 Ludwigshafen (Deutschland)

E-Mail: henrique.teles@basf.com

(2) Die Identifikationsnummern (ORCIDs) der Autoren sind unter

(iD) http://dx.doi.org/10.1002/ange.201604656 zu finden. stoffatome auf das Metall zu übertragen. Aber erst jetzt ist die Zeit gekommen, in der goldhaltige Heterogenkatalysatoren ihre Anwendung in großtechnischen Verfahren finden.

Wie man angesichts der edlen Natur des Metalls erwarten würde, sind Goldoberflächen nicht in der Lage, Sauerstoff zu aktivieren, und sie korrodieren auch nicht. Es war also eine große Überraschung, als gezeigt wurde, dass Gold in Form von Nanopartikeln durchaus ein exzellenter Katalysator sein kann. Diese Entdeckung markiert den Startschuss für die Jagd nach Goldheterogenkatalysatoren. Weiteren Vorschub erhielt dieses Forschungsgebiet, als kurz danach auch die Entdeckung folgte, dass Gold ebenfalls sehr aktive Homogenkatalysatoren bilden kann. ${ }^{[5]}$ Dies kulminiert in der Entdeckung, dass quasi-homogene Goldcluster, mit 3-10 Goldatomen, die in Lösung generiert werden können, Wechselzahlen von bis $\mathrm{zu} 10^{7}$ bei Raumtemperatur erreichen können. ${ }^{[6]}$ Alle diese Entdeckungen waren maßgeblich dafür verantwortlich, dass die Goldkatalyse, sowohl homogen als auch heterogen, zu dem lebhaften Forschungsgebiet wurde, das sie heute ist. ${ }^{[7,8]}$

Die Geschichte der Entdeckung, Entwicklung und Kommerzialisierung von Goldkatalysatoren für die Hydrochlorierung von Acetylen als Ersatz für die bisher verwendeten $\mathrm{HgCl}_{2}$-Katalysatoren wurde kürzlich zusammengefasst. ${ }^{[9]}$ Nach unserem Wissen gibt es bisher nur zwei goldhaltige Katalysatoren, die kommerziell angewendet werden: den Audotierten Pd-Katalysator für die Vinylacetat-Synthese, der schon seit fast einem halben Jahrhundert verwendet wird, ${ }^{[10]}$ und den Kern/Schale-AuNiO ${ }_{x}$-Katalysator für die oxidative Veresterung von Methacrolein zu Methylmethacrylat. ${ }^{[11]}$

Eine erste, 2005 publizierte Bewertung der kommerziellen Aspekte der heterogenen Goldkatalyse kam $\mathrm{zu}$ dem Schluss, dass die ungenügende Standzeit und das Fehlen einer praktikablen Methode für die Katalysatorherstellung die beiden Faktoren sind, die einer großtechnischen Anwendung im Wege stehen. ${ }^{[12]}$ Zudem muss ein neuer Katalysator immer im Kontext des Verfahrens gesehen werden, für das er ein- 
gesetzt wird ${ }^{[13]}$ Damit ein neuer Katalysator Erfolg hat, muss eine von zwei Bedingungen erfüllt werden: Ein neuer Katalysator wird dann erfolgreich, wenn er die Entwicklung eines neuen Verfahrens mit deutlich günstigeren Ausgangsverbindungen oder mit deutlich geringeren Investitionskosten ermöglicht. Dies war der Fall für den Katalysator der Vinylacetatsynthese, der einen Wechsel von der bisher verwendeten Rohstoffbasis Acetylen zum viel günstigeren Ethylen ermöglichte. In einem solchen Fall wird der neue Katalysator zum Bau von vielen neuen Anlagen und insgesamt zu einem Technologiesprung führen.

Auch wenn die Ausgangsstoffe nicht verändert werden, kann ein neuer Katalysator eine - meist bescheidene Besserstellung bieten, wenn er z.B. die Selektivität der Reaktion erhöht. In solchen Fällen wird der neue Katalysator in

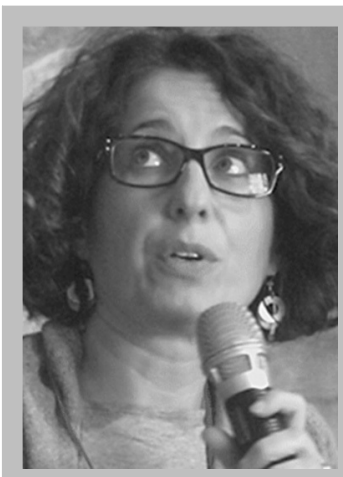

Rosaria Ciriminna studierte Chemie (Abschluss 1995 mit einer Arbeit über pflanzliche Naturstoffe) bei Prof. F. Piozzi an der University of Palermo, wo sie zunächst als chemisch-technische Assistentin angestellt worden war. In den Folgejahren forschte sie in Padua (Italien), Montpellier (Frankreich), Reading (Großbritannien) und Québec (Kanada). Sie ist seit 2001 Forschungschemikerin am in Palermo ansässigen Institute of Nanostructured Materials des italienischen Nationalen Forschungsrats (CNR), gegründet von Prof. G. Deganello. Ihre Forschungsgebiete sind Naturstoffe, Katalyse sowie grüne Chemie und Nanochemie.

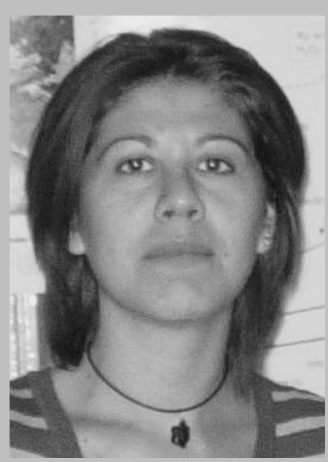

Ermelinda Falletta erhielt ihren M.Sc. in Chemie von der University of Palermo (Italien) und promovierte 2014 in industrieller Chemie an der University of Milan (Italien) bei Prof. L. Prati und C. Della Pina. Dort ist sie zurzeit als wissenschaftliche Mitarbeiterin tätig. Ihre Forschungsinteressen sind die Oxidation organischer Verbindungen durch $\mathrm{He}$ terogenkatalysatoren, grüne Synthese sowie innovative Anwendungen leitfähiger Polymere.

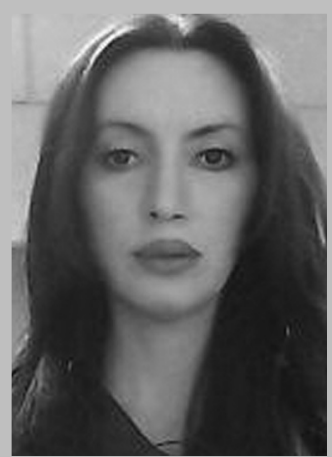

Cristina Della Pina erhielt ihren M.Sc. und Doktortitel in industrieller Chemie von der University of Milan (Italien) für Arbeiten unter Anleitung von Prof. M. Rossi. Derzeit ist sie Assistant Professor für allgemeine und anorganische Chemie an der University of Milan. Vor kurzem wurde ihr die National Scientific Qualification zum Associate Professor zuerkannt. Ihre Forschungsarbeiten konzentrieren sich auf die Entwicklung und Anwendung von Heterogenkatalysatoren zur selektiven Oxidation organischer Verbindungen und leitfähiger Polymere. der Regel nur zur Anwendung kommen, wenn er als Drop-in in einer vorhandenen Anlage eingesetzt werden kann, wie es sowohl für den Katalysator der Acetylen-Hydrochlorierung als auch für die oxidative Veresterung von Methacrolein der Fall war.

Gegenüber anderen Katalysatoren, die auf Metallen der Pt-Gruppe basieren,kann Gold für bestimmte Reaktionen eine signifikant höhere Aktivität und Selektivität aufweisen, und dies häufig sogar unter milderen Reaktionsbedingungen - ein Umstand, der durchaus den Weg zu neuen Anwendungen ebnen kann. ${ }^{[14]}$

Katalysatoren, die ähnlich teure trägerfixierte Metalle aus der Platingruppe enthalten, finden in der chemischen Industrie breite Anwendung, da sie in der Lage sind, für die Industrie wichtige Kleinmoleküle wie $\mathrm{H}_{2}$ oder $\mathrm{O}_{2}$ als die wichtigsten Reduktions- bzw. Oxidationsmittel aber auch CO, Olefine und andere grundlegende Bausteine der chemischen Industrie zu aktivieren. Die Tatsache, dass Gold auf der Erde sogar häufiger vorkommt als andere Metalle aus der Platingruppe, macht Gold noch attraktiver. So wurde für das Jahr 2015 die globale Goldproduktion aus dem Bergbau auf 3000 Tonnen geschätzt, ${ }^{[15]}$ während im gleichen Jahr die produzierte Menge an Pt nur 178 Tonnen und die an Pd nur 208 Tonnen betrug.

In den folgenden Abschnitten werden wir versuchen, die wichtigsten Hindernisse aufzuzeigen, die auf dem langen Weg von der Entdeckung hin zur kommerziellen Nutzung der Goldkatalyse überwunden werden mussten. Diese kritische Analyse soll gleichermaßen Forscher aus dem akademischen

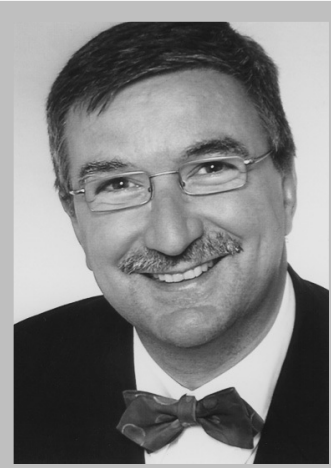

Joaquim Henrique Teles studierte Chemie und Verfahrenstechnik an der Technical University of Lisbon (Portugal) und promovierte 1988 an der Universität Gießen (Deutschland) unter Anleitung von Prof. G. Maier. Er war Postdoktorand bei Prof. W. von E. Doering an der Harvard University und später Humboldt-Stipendiat an der Universität Gießen, bevor er 1991 als Forschungschemiker zur BASF ging. Seit 2001 leitet er eine Forschungsgruppe im Bereich Process Research and Chemical Engineering der BASF in Ludwigshafen. Seine Forschung konzentriert sich auf Oxidationen (z. B. zur Produktion von Propylenoxid mit $\mathrm{H}_{2} \mathrm{O}_{2}$ und von $\left(y\right.$ clododecanon mit $\mathrm{N}_{2} \mathrm{O}$ ) und Phosgenchemie.

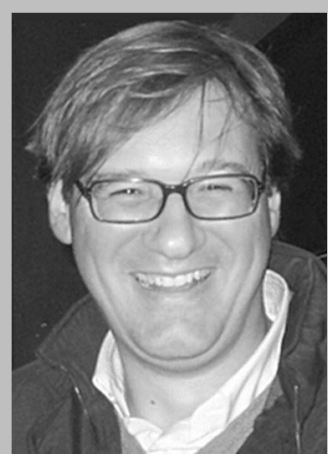

Mario Pagliaro studierte Chemie (Diplom 1993) an der University of Palermo und promovierte 1998 in Bologna mit einer Arbeit über katalytische Kohlenhydratoxidation, die hauptsächlich in der Gruppe von Prof. D. Aunir an der Hebrew University of Jerusalem durchgeführt worden war. Er ging dann in die Niederlande, wo er zunächst an der Leiden University und anschließend am Zeist's TNO Institute of Food Sciences bei Dr. A. de Nooy forschte. Im Jahr 2000 wurde er Wissenschaftler am chemischen Institut des italienischen Nationalen

Forschungsrats (CNR) in Palermo. Seine Forschung umfasst grüne und organische Chemie, Material- und Nanochemie, Solarenergie und Bioökonomie. 
und industriellen Umfeld leiten und inspirieren, die sich mit der Entwicklung von neuen Goldkatalysatoren oder neuen Gold-katalysierten Verfahren beschäftigen. Als Beispiele genannt seien hier die direkte Synthese von Wasserstoffperoxid aus den Elementen, ${ }^{[16]}$ die selektive Oxidation von Kohlenwasserstoffen ${ }^{[17]}$ und die selektive Oxidation von erneuerbaren Bausteinen, die im Rahmen der aufkommenden Bioraffinerien demnächst verfügbar werden könnten. ${ }^{[18]}$

\section{Oxidative Veresterung von Methacrolein zu Methylmethacrylat}

Seit 1976 produziert Asahi Kasei (früher Asahi Chemical) Methylmethacrylat in einer Anlage in Kawasaki (Japan). Diese Anlage verwendete zunächst ein dreistufiges Verfahren ausgehend von tert-Butylalkohol. Dieser wurde in der ersten Stufe in der Gasphase zu Methacrolein oxidiert und das Methacrolein in der nächsten Stufe, ebenfalls in der Gasphase, zu Methacrylsäure oxidiert, jeweils an zwei unterschiedlichen Mo-Katalysatoren. Die Methacrylsäure wurde zum Schluss mit Methanol verestert, um das gewünschte Methylmethacrylat zu bilden. Dieses Verfahren hatte etliche Nachteile, z.B. die hohen Investitionskosten für die drei Stufen und die geringe Standzeit des Katalysators für die zweite Stufe.

Um diese Probleme zu umgehen, entwickelte Asahi ein neues Verfahren, das so genannte Direct-Metha-Verfahren, in dem Acrolein in Gegenwart von Methanol in flüssiger Phase direkt zu Methylmethacrylat oxidativ verestert wird (Schema 1). ${ }^{[19]}$ Ursprünglich wurde die oxidative Veresterung

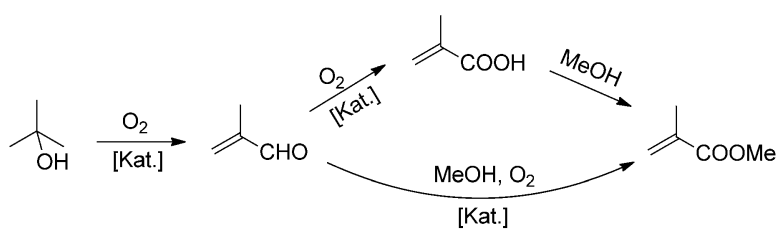

Schema 1. Das alte Asahi-Verfahren (obere Route) und das neue Direct-Metha-Verfahren (untere Route) für die Synthese von Methylmethacrylat.

von Methacrolein mit Methanol in Gegenwart von molekularem Sauerstoff mit einem Aluminiumoxid-fixierten Katalysator durchgeführt, wobei die intermetallische $\mathrm{Phase} \mathrm{Pd}_{3} \mathrm{~Pb}$ die Aktivphase war. ${ }^{[20]}$ Die Einführung der oxidativen Veresterung war eine sehr wichtige Entwicklung, die es ermöglichte, die Anzahl der Stufen von drei auf zwei zu verringern, wodurch die hohen Investitionskosten, als eines der wichtigsten Probleme des ursprünglichen Verfahrens, adressiert wurden. Ungeachtet dessen hatte das Direct-Metha-Verfahren, das seit 1982 im Einsatz war, noch immer Nachteile. Die Selektivität für Methylmethacrylat auf Basis von Methacrolein war relativ gut, jedoch oxidierte der Katalysator auch recht große Mengen an Methanol zu Methylformiat (pro mol Methylmethacrylat wurden $0.2 \mathrm{~mol}$ Methylformiat erzeugt). Hinzu kam noch, dass der $\mathrm{Pd}_{3} \mathrm{~Pb}$-Katalysator nicht einfach $\mathrm{zu}$ produzieren war und $\mathrm{Pd}$, das nicht in der $\mathrm{Pd}_{3} \mathrm{~Pb}$-Phase ge- bunden war, zu Selektivitätsverlusten durch die Decarbonylierung von Methacrolein führte, wobei die drängendsten Problem ganz gewiss die Verringerung der Methanolverluste sowie das Vermeiden der Bildung von Methylformiat als Nebenprodukt waren. Dem Asahi-Forschungsteam um Suzuki gelang die Entdeckung eines neuen Katalysators, der den ursprünglichen $\mathrm{Pd}_{3} \mathrm{~Pb}$-Katalysator ersetzen konnte und eine viel niedrigere Bildungsgeschwindigkeit für Methylformiat aufwies.

Seit den Pionierarbeiten von Hayashi und seiner Gruppe bei Nippon Shokubai zwischen 2002 und 2006 über die oxidative Veresterung von Ethylenglycol mit Methanol zu Methylglycolat war bekannt, dass sich Goldkatalysatoren gut für derartige oxidative Veresterungen eignen; ${ }^{[21]}$ Gold war das bevorzugte Metall, manchmal noch mit $\mathrm{Pb}$ als Dotierungselement, besonders wenn es auf Aluminiumoxid fixiert war. Obwohl die Selektivität bezogen auf Ethylenglycol sehr gut war, produzierten diese Katalysatoren je nach dem Verhältnis von Methanol zu Ethylenglycol auch beachtliche Mengen von Methylformiat als Nebenprodukt (zwischen 0.15 und $0.36 \mathrm{~mol}$ Methylformiat pro mol Methylglycolat). Die Arbeiten bei Nippon Shokubai gingen nie über den Pilotmaßstab hinaus, aber sie lieferten sicher die Inspiration für die Gruppe bei Asahi Kasei.

Der beste Katalysator, der von den Forschern bei Asahi Kasei identifiziert werden konnte, war ein $\mathrm{Au}-\mathrm{NiO}_{x} / \mathrm{SiO}_{2}-$ $\mathrm{Al}_{2} \mathrm{O}_{3}-\mathrm{MgO}$-Katalysator, der in der Lage war, Methylmethacrylat mit einer Selektivität von $98 \%$ bezogen auf Methacrolein bei einem Umsatz von $58 \%$ zu liefern. Bahnbrechend war dabei, dass dieser Katalysator nur $0.007 \mathrm{~mol}$ Methylformiat pro mol Methylmethacrylat bildete. Leider war der im Labor entdeckte Katalysator noch nicht hinreichend mechanisch stabil für eine technische Verwendung.

Um dieses Problem zu lösen, entwickelte das Team bei Asahi Kasei einen Kern/Schale-Katalysator, bei dem Au-Nickeloxid-Nanopartikel $\left(\mathrm{AuNiO}_{x}\right)$ auf einem Siliciumoxid-basierten Träger immobilisiert sind, wobei sich die Goldnanopartikel etwas unterhalb der äußeren Oberfläche befinden und so vor Abtragung durch Abrasion geschützt sind. Die Stabilität dieses Katalysators wurde in Langzeitexperimenten in einer Pilotanlage verifiziert, und schließlich wurde der Katalysator 2008 in der bereits existierenden kommerziellen MMA-Produktionsanlage eingesetzt. ${ }^{[22]}$

Die $\mathrm{AuNiO}_{\mathrm{x}}-\mathrm{Nanopartikel,} \mathrm{die} \mathrm{bevorzugt} 20 \mathrm{Mol}-\% \mathrm{Au}$ enthalten, haben eine Kern/Schale-Struktur, mit Au-Nanopartikeln im Kern und hochoxidiertem $\mathrm{NiO}_{x}$ an der Oberfläche. Diese Kern/Schale-Nanopartikel waren sehr aktiv, aber noch nicht technisch einsetzbar, da die oxidative Veresterung von Methacrolein in Suspension stattfindet und der Katalysator somit einer hohen mechanischen Beanspruchung ausgesetzt ist. Die Abrasion, die durch den mechanischen Stress verursacht wird, führte zu einem Verlust an Aktivphase und zur Desaktivierung des Katalysators.

Zur Lösung dieses Problems verwendete das Asahi-Team einen Träger aus einem mechanisch robusten Mischoxid, $\mathrm{SiO}_{2}-\mathrm{Al}_{2} \mathrm{O}_{3}-\mathrm{MgO}$, in Form von $60 \mu \mathrm{m}$ großen Kugeln und steuerte den Abscheidungsprozess derart, dass die Au-Nanopartikel bevorzugt knapp unter der Oberfläche bis zu einer Tiefe von $10 \mu \mathrm{m}$ abgeschieden wurden (Abbildung 1). Auf 


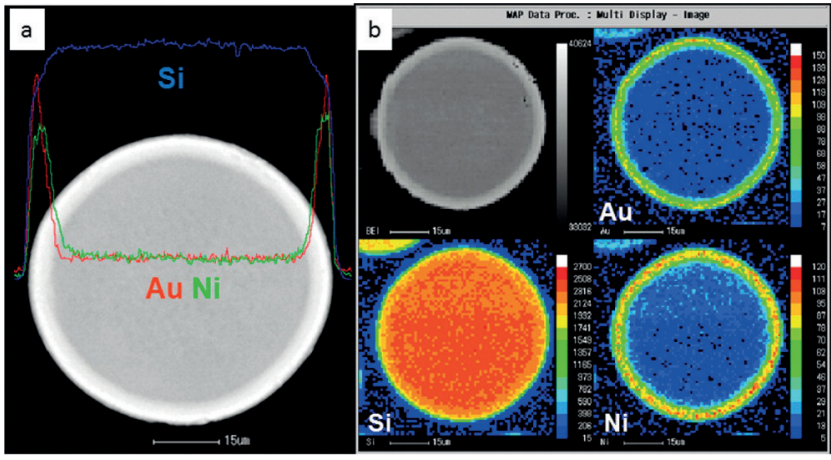

Abbildung 1. Elektronenstrahlmikroanalyse eines einzelnen Katalysatorkorns von $\mathrm{AuNiO}_{x} / \mathrm{SiO}_{2}-\mathrm{Al}_{2} \mathrm{O}_{3}-\mathrm{MgO}$. a) Sekundärelektronenbild und radiale Konzentrationsprofile. b) Farbkodierte Elementverteilungen ( $\mathrm{Ni}$ grün, Au rot) [Wiedergabe aus Lit. [11] mit Genehmigung].

diese Weise bleiben die Au-Nanopartikel für die Reaktanten gut zugänglich, die Verluste an Aktivphase durch Abrasion können aber minimiert werden. Nur durch diese spezielle Abscheidemethode war es möglich, einen Katalysator zu präparieren, der robust und langlebig war. Wie in Abbildung 2 gezeigt, wurde der Katalysator in einem kontinuierli-

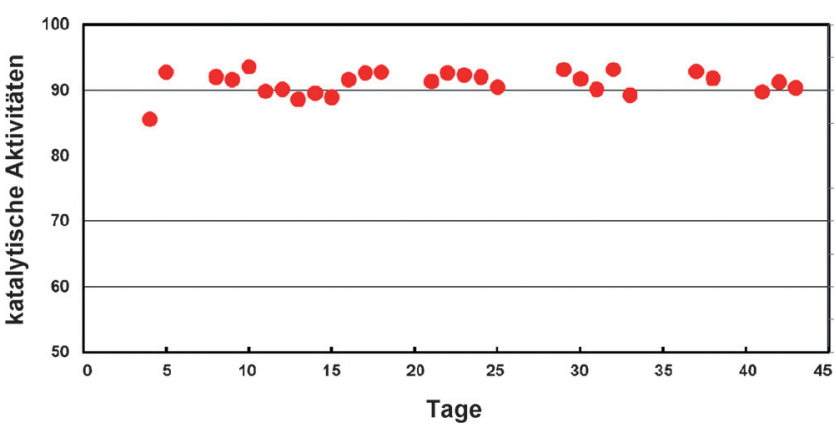

Abbildung 2. Katalytische Aktivität von $\mathrm{AuNiO} / \mathrm{SiO}-\mathrm{Al}_{2} \mathrm{O}_{3}-\mathrm{MgO}$ in $\mathrm{Ab}$ hängigkeit von der Versuchszeit während des kontinuierlichen Langzeittests [Wiedergabe aus Lit. [11] mit Genehmigung]

chen Reaktor ohne sichtbares Abfallen der katalytischen Aktivität über 40 Tage betrieben. Während der Testphase wurde Methylmethacrylat mit einer hohen Selektivität von 96-97\% bezogen auf Methacrolein und in einer konstanten Ausbeute von $60 \%$ erhalten. Das Auslaugen von Au und Ni war vernachlässigbar, und die Konzentration dieser beiden Elemente in der Reaktionsmischung betrug $<2.5 \mathrm{ppb}$. Eine Untersuchung des Katalysators nach dem Langzeittest zeigte kein Sintern der $\mathrm{AuNiO}_{x}$-Nanopartikel, und die Kern/SchaleStruktur blieb ebenfalls intakt.

Zusammengefasst waren verschiedene Faktoren für den Erfolg dieses Katalysators ausschlaggebend: Erstens waren die Reaktionsbedingungen für den neuen Katalysator sehr ähnlich zu denjenigen für den alten. Weiterhin hatte der neue Katalysator eine Desaktivierungsgeschwindigkeit (sowohl chemisch als auch mechanisch), die nicht größer als die des ursprünglichen Katalysators war. Dies waren die unverzichtbaren Bedingungen, die es zu erfüllen galt, damit der neue Katalysator in der vorhandenen Anlage eingesetzt werden konnte. Als Vorteil bietet der neue Katalysator eine verminderte Nebenprodukt-Bildungsgeschwindigkeit. Bei der Menge an gebildetem Abwasser gab es dagegen keine Änderung. Zusätzlich bringt der Katalysator niedrigere spezifische Kosten mit sich, da für die gleiche Produktivität weniger Edelmetall eingesetzt werden muss.

\section{Hydrochlorierung von Acetylen}

Der Katalysatorhersteller Johnson Matthey kündigte 2015 offiziell die Einführung einer neuen Katalysatormarke namens Pricat MFC an. Dieser neue Au-Katalysator wurde speziell für die Herstellung von Vinylchlorid-Monomer (VCM) entwickelt. VCM ist das Monomer für Polyvinylchlorid (PVC), den mengenmäßig drittbedeutendsten Kunststoff der Welt. ${ }^{[23]}$ Für etwa ein Drittel der 50 Millionen Jahrestonnen Weltkapazität von VCM wird eine Technik auf Basis der Hydrochlorierung von Acetylen genutzt, wobei diese Anlagen fast ausschließlich in China angesiedelt sind. Diese Anlagen verwenden als Katalysator $\mathrm{HgCl}_{2}$ auf Aktivkohle und sind deshalb unter einen besonderen regulatorischen Druck geraten. Gemäß des Minamata-QuecksilberÜbereinkommens müssen bis 2022 alle VCM-Anlagen auf einen Quecksilber-freien Katalysator umgestellt sein, vorausgesetzt ein solcher Katalysator ist verfügbar und die Alternative ist wirtschaftlich vertretbar. Der Quecksilberkatalysator hat eine sehr begrenzte Lebenszeit von nur sechs Monaten, und die Quecksilberverluste während des Betriebs sowie der Katalysatorrückgewinnung für alle existierenden Anlagen addieren sich auf 600 Tonnen pro Jahr, was in etwa der Hälfte des Weltverbrauches an Quecksilber entspricht.

Es gibt also sowohl aus regulatorischen Gründen wie auch aus Umweltschutzsicht große Anreize für den Ersatz des Quecksilberkatalysators durch neue Katalysatoren. Der neue Katalysator muss jedoch so beschaffen sein, dass er in den existierenden Anlagen eingesetzt werden kann, was daran liegt, dass er keinen großen wirtschaftlichen Schub anbieten können wird: Die Anlagen werden weiterhin die gleichen Einsatzstoffe verwenden, und es können nur noch marginale Selektivitätsverbesserungen erzielt werden. Der neue Katalysator muss also unter ähnlichen Reaktionsbedingungen arbeiten wie der alte, muss eine in etwa gleiche Aktivität und eine mindestens ebenbürtige Selektivität aufweisen, und die Katalysatorkosten müssen in der Größenordnung des zu ersetzenden Quecksilberkatalysators liegen.

Die katalytische Aktivität von Tetrachlorogoldsäure für die Addition von $\mathrm{HCl}$ an Acetylen, sowohl in homogener als auch in heterogener Form, war bereits seit 1977 bekannt, nachdem die japanische Firma Denki Kagaku Kogyo (heute als Denka bekannt) zwei Patente dazu angemeldet hatte. ${ }^{[24]}$ Die Patente beschäftigten sich in erster Linie mit der Verwendung von Gemischen aus Gold mit Palladium oder Platin, enthielten aber auch Vergleichsbeispiele, die zeigten, dass auch Gold alleine eine gute Aktivität und Selektivität aufwies. Leider gerieten diese Patente in Vergessenheit und es dauerte weitere zehn Jahre, bis dieses Thema von Hutchings und seiner Gruppe wieder aufgegriffen wurde. Hutchings und Joffe konnten zeigen, dass Tributylammoniumtetrachloroa- 
urat in einem Lösungsmittelgemisch aus 2-Propanol $/ n$-Decan ein (wenngleich nur mäßig) aktiver Homogenkatalysator für die Addition von $\mathrm{HCl}$ an Acetylen ist. ${ }^{[25]}$

Die ersten Heterogenkatalysatoren wurden durch simple Imprägnierung eines Aktivkohleträgers mit einer wässrigen $\mathrm{HAuCl}_{4}$-Lösung hergestellt. ${ }^{[3,26]}$ Diese Katalysatoren waren fast so aktiv und selektiv wie der Quecksilberkatalysator, wurden aber rasch desaktiviert. Zwar konnten die desaktivierten Au-Katalysatoren durch eine Behandlung mit $\mathrm{HCl}$ wieder regeneriert werden, jedoch verlief die Desaktivierung im zweiten Zyklus mindestens so schnell wie mit einem frischen Katalysator. Es war also klar, dass ein solcher Katalysator technisch nicht anwendbar wäre.

In den Folgejahren konnten zwei Desaktivierungsmechanismen identifiziert werden: Bei niedrigen Reaktionstemperaturen $\left(60-100^{\circ} \mathrm{C}\right)$ war die Verkokung des Katalysators der Hauptdesaktivierungsmechanismus; bei höheren Temperaturen dagegen $\left(120-180^{\circ} \mathrm{C}\right)$ war die Reduktion von katalytisch aktiven kationischen Goldspezies zu inaktivem $\mathrm{Au}^{0}$ der Hauptmechanismus. ${ }^{[27]}$ In der gleichen Studie zeigten die Autoren, dass eine Trägerfixierung des Golds in Gegenwart von Königswasser, das ein starkes Oxidationsmittel ist, deutlich aktivere Katalysatoren lieferte. Die Autoren führten dies auf ein vermehrtes Auftreten von kationischen Goldspezies auf der Oberfläche der deponierten Goldnanopartikel zurück. Trotz der hohen Goldbeladungen und der verbesserten Abscheidungsmethode konnte das Problem der Katalysatordesaktivierung jedoch nicht gelöst werden.

Um einen wirtschaftlich tragbaren Alternativkatalysator für die VCM-Produzenten anbieten zu können, musste das Team um Johnston bei Johnson Matthey bestimmte Randbedingungen festlegen. Hierzu gehörten eine maximale Goldbeladung von $0.25 \%$ sowie eine Katalysatorpräparation, bei der auf den Einsatz von Königswasser verzichtet werden konnte, um Schwierigkeiten mit der Entsorgung Königswasser-haltiger Abfallströme zu vermeiden. Die anvisierte Beladung war zwar signifikant geringer als die üblichen $0.5-1 \%$ von konventionellen $\mathrm{Au} / \mathrm{C}$-Katalysatoren, die in Gegenwart von Königswasser hergestellt wurden, aber das Hauptproblem, das es zu lösen galt, war die Katalysatordesaktivierung. ${ }^{[28]}$ Dies war erforderlich, um die anvisierten niedrigen Katalysatorkosten zu erreichen und so gegenüber dem $\mathrm{HgCl}_{2}-$ basierten Katalysator konkurrenzfähig zu sein.

Aus der Betrachtung der Katalysatorentwicklung lassen sich wertvolle Einsichten gewinnen. Bereits 1995 hatte Aker Solutions, ein norwegischer Chemieanlagenbauer, der später von Jacobs zugekauft wurde, einen Au-Katalysator für die VCM-Synthese entwickelt. Der Katalysator wurde im Pilotmaßstab getestet und zeigte dabei sogar eine höhere Lebensdauer als der Quecksilberkatalysator. ${ }^{[29]}$ Dieser Katalysator wurde jedoch nie kommerzialisiert, und das Projekt wurde gestoppt. Ab 2006 startete Jacobs dann eine Kooperation mit Johnson Matthey und mit der Gruppe von Hutchings in Cardiff, um einen an die Bedingungen des chinesischen VCM-Verfahrens angepassten $\mathrm{Au} / \mathrm{C}-$ Katalysator zu entwickeln. Schon ein Jahr später konnten Hutchings und Mitarbeiter zeigen, dass Gold alleine, ohne Zusatz von weiteren Metallen, die höchste katalytische Aktivität aufweist. Sie konnten auch zeigen, dass eine hohe Dispersion des
Goldes notwendig ist, um die Menge an katalytisch aktiven kationischen Goldspezies zu maximieren. ${ }^{[30]}$ Nachdem dies verstanden worden war, wurde die Präparation des Katalysators zum bestimmenden Hebel und zugleich zur größten Herausforderung.

2010 erhielt Jacobs den Auftrag, eine Pilotanlage zu errichten, die an die Anlage eines chinesischen VCM-Produzenten angegliedert war. Diese Seitenstromanlage hatte ein Reaktionsrohr mit den gleichen Abmessungen (3 m Länge, mit $2 \mathrm{~kg}$ Katalysator beladen) wie ein einzelnes Rohr des technisch verwendeten Rohrbündelreaktors. ${ }^{[31]}$ Diese Pilotanlage wurde ab Mitte 2011 kontinuierlich über zwei Jahre betrieben. In dieser Zeit wurden sechs Katalysatoren getestet, um den Katalysator im Detail zu optimieren. Am Ende gelang es, einen hochaktiven Katalysator mit nur $0.1 \% \mathrm{Au} \mathrm{zu}$ entwickeln, indem $\mathrm{Na}_{3} \mathrm{Au}\left(\mathrm{S}_{2} \mathrm{O}_{3}\right)_{2}$ auf Aktivkohleextrudate fixiert wurde. ${ }^{[32]}$ Offensichtlich trägt das schwefelhaltige Anion entscheidend dazu bei, die kationischen Goldspezies zu stabilisieren. Erst deren Verwendung ermöglichte die Herstellung eines Katalysators, der weniger desaktivierungsanfällig ist, ohne dass der Einsatz eines starken Oxidationsmittels wie Königswasser notwendig ist.

Die Tests belegten, dass diese Familie von Au/C-Katalysatoren hochaktiv und hochselektiv ist. Der abschließende Test dauerte neun Monate, obwohl bereits nach sechs Monaten von einem erfolgreichen Test gesprochen werden konnte. Nach diesem Test wurde 2012 ein Betriebsversuch anberaumt, bei dem ein kommerzieller Produktionsreaktor mit 790 Reaktionsrohren mit insgesamt 1.6 Tonnen des Goldkatalysators beschickt wurde. Dieser Reaktor ist seit 2013 in Betrieb.

Der Rohrbündelreaktor wurde, unter den gleichen Bedingungen wie mit dem $\mathrm{HgCl}_{2} / \mathrm{C}$-Katalysator, mehr als $4500 \mathrm{~h}$ betrieben (Abbildung 3). Bis zu einer Ausbeute von $1000 \mathrm{~kg}$ VCM pro kg Katalysator zeigte der Au-Katalysator die glei-

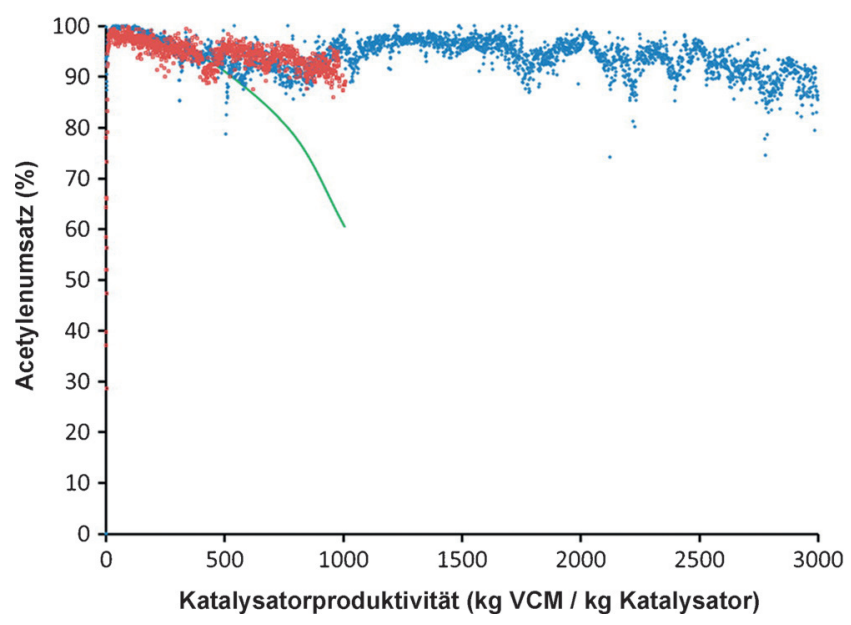

Abbildung 3. Vergleich der Leistung des $0.1 \% \mathrm{Au} / \mathrm{C}-$ Katalysators im Pilotbetrieb (rote Linie, bis zu einer Ausbeute von $1000 \mathrm{~kg}$ VCM pro kg Katalysator) und im Produktionsreaktor (blaue Linie, bis zu eine Ausbeute von 3000 kg VCM pro kg Katalysator) mit der eines konventionellen $10 \% \mathrm{HgCl}_{2} / \mathrm{C}$-Katalysators (grüne Linie). Katalysator $=0.1 \% \mathrm{Au} / \mathrm{C}$, hergestellt durch Fixierung von $\mathrm{Na}_{3} \mathrm{Au}\left(\mathrm{S}_{2} \mathrm{O}_{3}\right)_{2}$ auf Aktivkohleextrudaten [Wiedergabe aus Lit. [9] mit Genehmigung]. 
che gute Leistung wie im Pilotversuch, was bereits deutlich besser als die mit einem $\mathrm{HgCl}_{2} / \mathrm{C}$-Katalysator erreichbare Ausbeute ist. Der Au-Katalysator erzielt aber Ausbeuten, die weit über denjenigen liegen, die mit dem Quecksilberkatalysator möglich wären, und zeigt dabei immer noch gute Aktivität zu einem Zeitpunkt, an dem der konventionelle Katalysator schon längst hätte ausgetauscht werden müssen.

Auch in diesem Fall war die erfolgreiche Einführung des Goldkatalysators nur möglich, weil er sich im bestehenden Verfahren einsetzen ließ, ohne dass die vorhandenen Anlagen umgebaut werden mussten - und natürlich auch, weil sich die Gesamtwirtschaftlichkeit des Verfahrens, bei gleichzeitiger Eliminierung der Quecksilberemissionen, verbessern ließ.

\section{Produktion von industriellen Goldkatalysatoren}

Goldheterogenkatalysatoren auf verschiedenen Trägern werden bereits von einigen Firmen hergestellt und kommerziell angeboten. ${ }^{[33]}$ Die südafrikanische AuTEK, ein Gemeinschaftsunternehmen von Mintek und drei Minengesellschaften, war die erste Firma, die 2009 eine Produktionsanlage zur Synthese technischer Mengen von Katalysatoren mit $1 \% \mathrm{Au}$ in reproduzierbarer Qualität errichtete. Diese Katalysatoren wurden unter den geschützten Markennamen AUROlite $\left(\mathrm{Au} / \mathrm{TiO}_{2}, \mathrm{Au} / \mathrm{ZnO}, \mathrm{Au} / \mathrm{Al}_{2} \mathrm{O}_{3}\right)$ und AUROlith (AU/ $\mathrm{Al}_{2} \mathrm{O}_{3} /$ Cordierit) in den Markt eingeführt und wurden nach der 2005 zum Patent angemeldeten Methode der Abscheidung-Fällung hergestellt. ${ }^{[34]}$ Sie wurden insbesondere für Anwendungen wie Oxidationsreaktionen, sowohl in der Gasphase als auch in flüssiger Phase, vermarktet.

Ein weiterer kommerzieller Au-Katalysator, der von $3 \mathrm{M}$ speziell als CO-Oxidationskatalysator in Fluchtmasken entwickelt wurde, ist NanAucat. Er besteht aus trägerfixierten Au-Nanopartikeln, die durch chemische Abscheidung aus der Gasphase auf poröse, amorphe Kohle aufgebracht werden. ${ }^{[35]}$ Der Katalysator wird als der aktivste und stabilste Katalysator für die oxidative CO-Entfernung vermarktet. In der Tat ist NanAucat unempfindlich gegen Feuchtigkeit, was der größte Nachteil des bisher üblichen Katalysators Hopcalit ist. Deshalb benötigt der Goldkatalysator kein vorgeschaltetes Trocknungsbett, was zu einer großen Gewichtseinsparung führt. Der Katalysator ist jedoch immer noch empfindlich gegen eine Vergiftung durch Amine oder Schwefelverbindungen.

Im Allgemeinen, und wie bereits in der Einleitung erwähnt, sind die Hauptprobleme, die bereits 2005 identifiziert und diskutiert wurden, die Einstellung der Größe der AuNanopartikel und die Reproduzierbarkeit der Synthesemethode. ${ }^{[12]}$ Jüngere Entwicklungen auf dem Gebiet der Katalysatorpräparation haben viele dieser Probleme gelöst. Anders als in der Vergangenheit sind heute hochaktive und selektive Katalysatoren mit niedriger Au-Beladung auf reproduzierbare Art und Weise herstellbar.

Beispielsweise bestand eines der Erfolgskriterien, die zur industriellen Anwendung eines Au-Katalysators in der VCMSynthese führten, in der Fähigkeit, ausreichend aktive AuKatalysatoren mit nur $0.25 \%$ Au-Beladung (statt der sonst üblichen $1 \%$ Au-Beladung der Katalysatoren der ersten
Generation) herzustellen. Die Route, die von Johnston und Mitarbeitern entwickelt wurde, basiert auf einer neuen Methode, mit der kationische Goldspezies gezielt stabilisiert werden können. Die Methode ermöglicht es, Katalysatoren mit Beladungen zwischen 0.15 und $0.6 \%$ Au ausgehend von schwefelhaltigen Au-Komplexen in wässriger Lösung herzustellen. Dabei stabilisiert Schwefel die gewünschten kationischen Goldspezies.

Im Fall des Asahi-Katalysators wird als Träger ein $\mathrm{SiO}_{2}{ }^{-}$ $\mathrm{Al}_{2} \mathrm{O}_{3}-\mathrm{MgO}-\mathrm{Mischoxid}$ verwendet, das nach einem Sol-GelVerfahren hergestellt wird. Der Träger liegt in Form von 60$\mu \mathrm{m}$-Kugeln vor, hat eine Oberfläche von $149 \mathrm{~m}^{2} \mathrm{~g}^{-1}$ und einen mittleren Porenradius von $8 \mathrm{~nm}$. Auf diesem Träger werden dann durch Cofällung $\mathrm{Au}$ und $\mathrm{NiO}$ abgeschieden. Dafür verwendet man eine wässrige Lösung von $\mathrm{Ni}\left(\mathrm{NO}_{3}\right)_{2} \cdot 6 \mathrm{H}_{2} \mathrm{O}$ und $\mathrm{HAuCl}_{4}\left(1.3 \mathrm{molL}^{-1}\right)$, die bei $90^{\circ} \mathrm{C}$ auf dem Träger imprägniert wird. Anschließend wird im Vakuum getrocknet und $5 \mathrm{~h}$ bei $450^{\circ} \mathrm{C}$ kalziniert. Der Katalysator wird als braunes Pulver erhalten und enthält 0.9 Gew.- $\%$ Au sowie 1.1 Gew.-\% Ni.

\section{Heterogene gegenüber homogener Katalyse}

Bemerkenswerterweise sind die ersten technischen Anwendungen von Goldkatalysatoren allesamt heterogen katalysierte Verfahren für großvolumige Grundchemikalien und nicht, wie man vielleicht erwarten würde, für Feinchemikalien oder Pharmawirkstoffe. Dies überrascht umso mehr, wenn man die sehr geringe Giftigkeit von Gold gegenüber der anderer Metalle aus der Platingruppe bedenkt, was besonders für Anwendungen in der Synthese von Pharmawirkstoffen ein großer Vorteil sein könnte. Auf diesem besonders streng regulierten Arbeitsgebiet sind die Restgehalte von Palladium oder Platin (beide mittlerweile als ,metals of significant safety concern" klassifiziert) in Arzneistoffen auf unter 10 ppm, wenn die Arznei oral verabreicht wird, oder sogar $<1$ ppm, wenn sie parenteral verabreicht wird, begrenzt worden. Aber auch dieser Umstand hat dem Gold bisher nicht zu einem Durchbruch verholfen. ${ }^{[36]}$

Die hohen Kosten von Gold machen eine möglichst vollständige Wiedergewinnung des Metalls zwingend erforderlich, z.B. mit einem Thiol-funktionalisierten Kieselgel. Die Verwendung von solchen Scavengers bringt allerdings zusätzliche Kosten für dieses Reagens mit sich und zerstört darüber hinaus auch den Goldhomogenkatalysator. Dies mag mit ein Grund sein, warum Goldhomogenkatalysatoren bisher noch keine industrielle Anwendung gefunden haben, auch wenn die Palette an Reaktionen, die durch Gold katalysiert werden, mit jedem Tag wächst. ${ }^{[37]}$

Das Potential von Goldhomogenkatalysatoren in der selektiven elektrophilen Aktivierung von $\mathrm{C} \equiv \mathrm{C}$-Bindungen unter milden Bedingungen wurde bereits 1997 erkannt. Forscher bei der BASF beschrieben zum ersten Mal einen kationischen $[\mathrm{L}-\mathrm{Au}]^{+}$-Komplex (wobei L ein Phosphin- oder Arsinligand ist), der in der Lage ist, die Addition von Alkoholen an Alkine unter milden Bedingungen (293-323 K) mit beeindruckenden Wechselzahlen von bis zu $5400 \mathrm{~h}^{-1}$ zu katalysieren. ${ }^{[38]}$ Diese hohe katalytische Aktivität lag um einige Größenord- 
nungen über denjenigen, die mit einfachen Gold(III)-Salzen erreichbar waren (wie von Utimoto und Fukuda beschrieben) ${ }^{[39]}$ Aber auch solch hohe katalytische Aktivitäten sind kein Garant für eine erfolgreiche technische Anwendung. Die Forscher bei BASF suchten damals nach einem Katalysator für die Synthese von 2,2-Dimethoxypropan (ein Baustein für die Synthese von Vitamin E) ausgehend von einem Gemisch aus Propin und Allen (das als Nebenprodukt beim Steamcracking von Naphtha verfügbar ist). Die Goldkomplexe waren zwar viel aktiver und langlebiger als die bisher bekannten Quecksilberkatalysatoren, hatten aber den Nachteil, dass nur Propin mit einer akzeptablen Geschwindigkeit abreagierte, während Allen weitgehend unumgesetzt blieb. Schließlich erwies sich ein einfacher Katalysator auf Basis eines Zinksilicats als deutlich bessere Alternative, nicht nur wegen der sehr geringen Katalysatorkosten, sondern auch, weil Propin und Allen mit ähnlichen Geschwindigkeiten umgesetzt wurden. ${ }^{[40]}$

Im Fall von Pd konnten Ananikov und Kashin zeigen, dass sowohl homogene als auch heterogene Katalyse zur Produktbildung beitragen können, indem durch Auslaugung aus Pd-Nanopartikeln sowohl homogene Pd-Komplexe als auch Cluster in Lösung gebildet werden. ${ }^{[41]}$ Ein ähnliches Phänomen ist im Fall von Goldheterogenkatalysatoren ebenfalls denkbar. Dies eröffnet Perspektiven für möglicherweise technisch relevante Anwendungen, indem man das Randgebiet zwischen heterogener und homogener Katalyse gezielt nutzt. Ein Beispiel für eine solche gezielte Anwendung wurde 2012 von Toste und Mitarbeitern mit Dendrimer-verkapseltem Gold gezeigt. ${ }^{[42]}$ Durch Modifizierung des Dendrimers konnte die katalytische Aktivität in einer Art und Weise, die an die Feinabstimmung von Liganden in der homogenen Katalyse erinnert, gezielt verändert werden. Ausgehend von Untersuchungen zur Fixierung von Goldhomogenkatalysatoren an Aminopropylsiloxy-funktionalisierte Siliciumoxidoberflächen entdeckten Hashmi und Mitarbeiter den bisher aktivsten Goldkatalysator. ${ }^{[43]}$ In diesem einkernigen Katalysator ist $\mathrm{Au}$ an einen Phosphorliganden mit sterisch hoch anspruchsvollen Substituenten gebunden, und dieser sterische Anspruch wird als Grund für die langsame Desaktivierung und dadurch besonders hohe Langlebigkeit des Katalysators genannt. ${ }^{[44]}$

Im Rahmen von Studien zur CO-Oxidation an $\mathrm{Au} / \mathrm{TiO}_{2-}$ und $\mathrm{Au} / \mathrm{Fe}_{2} \mathrm{O}_{3}$-Katalysatoren konnten Klyushin et al. zeigen, dass die Wechselwirkung zwischen Au-Nanopartikel und Träger eine sehr wichtige Rolle spielt und sogar wichtiger sein kann als eine Verringerung der Nanopartikelgröße. In der Kontaktzone zwischen Träger und Goldnanopartikel bildet sich eine Überlappungsschicht, die einen großen Einfluss auf die elektronische Struktur der Au-Nanopartikel sowie auf den Ladungstransfer zu und die Stabilisierung von niedrig koordinierten Au-Atome(n) im Goldnanopartikel haben kann (Abbildung 4). ${ }^{[45]}$

Gerade bei der Katalyse mit Nanopartikeln sind Defekte im Kristallgitter (wie Ecken, Kanten, Stufen, Verzwillingungen oder niedrig koordinierte Atome) vermutlich die katalytisch aktiven Stellen. Folgerichtig sind jedwede MetallTräger-Wechselwirkungen, die zur Stabilisierung von solchen

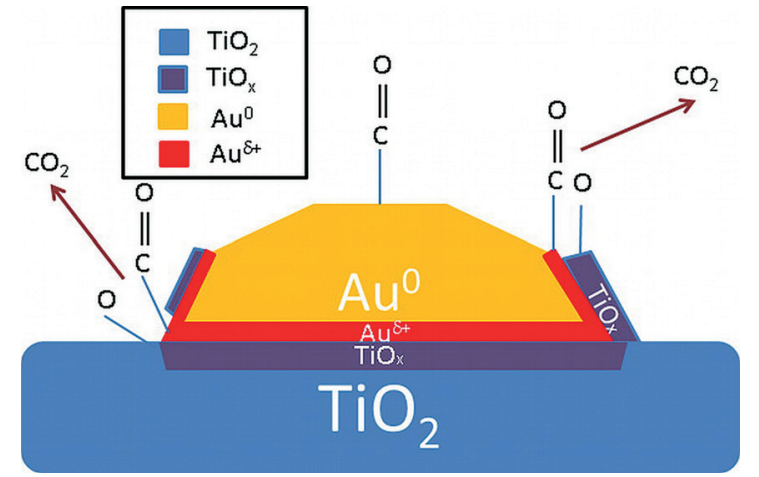

Abbildung 4. Postulierter Mechanismus der CO-Oxidation an $\mathrm{Au} / \mathrm{TiO}_{2}$ [Wiedergabe aus Lit. [45] mit Genehmigung]

Oberflächendefekten beitragen, förderlich für die katalytische Aktivität.

\section{Ausblick}

1976 äußerte Sermon die Ansicht, dass Gold „ein außergewöhnlich guter Katalysator" sei ${ }^{[46]}$ Fast vierzig Jahre später startete Asahi Kasei die erste technische Verwendung eines Goldkatalysators für die Methylmethacrylat-Produktion in Kawasaki (Japan), dicht gefolgt von einem PVC-Hersteller in der Nähe von Shanghai, der als erster von einem Quecksilberauf einen Goldkatalysator umgestellt hat. Werden diesen ersten großtechnischen Anwendungen andere folgen? Wird Gold das ihm prophezeite „Potential, Leben zu retten, die Gesundheit der Menschheit zu verbessern und den Planet zu säubern" erfüllen ? ${ }^{[47]}$

Um die Tragweite dieser neuen Entwicklungen zu begreifen, müssen wir uns vergegenwärtigen, dass alleine China für $50 \%$ der Quecksilberemissionen auf der ganzen Welt verantwortlich ist und $60 \%$ davon auf die PVC-Anlagen zurückzuführen sind. Die Kapazität für die Herstellung des benötigten $\mathrm{HgCl}_{2}$-Katalysators beträgt in China mehr als 4000 Tonnen $\mathrm{Jahr}^{-1}$. $^{[4]}$ Insbesondere weil Gold immer noch als sichere Investition in Krisenzeiten angesehen wird, stieg sein Preis nach der Finanzkrise 2008 kräftig an. Anfang März 2016 betrug der Preis für eine Unze Gold 1245 US\$, ${ }^{[49]}$ während die gleiche Menge an Palladium gerade einmal die Hälfte kostete (627 US\$). ${ }^{[50]}$ Noch ein paar Jahre zuvor, im

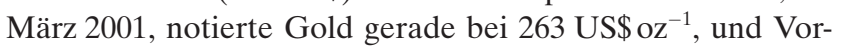
hersagen gingen davon aus, dass Gold eher durch einen stabilen Preis charakterisiert sein würde, im Unterschied zu den wild ausschlagenden Preisen für andere Metalle aus der Platingruppe ${ }^{[51]}$ Dies zeigt eindrücklich, wie schwer es ist, treffende Preisprognosen für Edelmetalle zu erstellen. Gerade Gold und andere Edelmetalle werden immer wieder Objekt von finanziellen Spekulationen sein, ${ }^{[52]}$ was zu starken Preisfluktuationen führen kann.

Dies hat bereits dazu geführt, dass anvisierte Anwendungen gescheitert sind. Ein Beispiel hierfür ist die Verwendung von Gold in Abgaskatalysatoren. Ziel der Entwicklungsarbeiten der Firma NanoStellar war damals, die Hälfte des $\mathrm{Pt} / \mathrm{Pd}$ in Diesel-Abgaskatalysatoren mit preiswerterem 
$\mathrm{Pt} / \mathrm{Au}$ zu ersetzen. Leider übersteig ab August 2011 der Preis für Gold den Preis für Platin, und das Geschäftsmodell von NanoStellar wurde damit unattraktiv. ${ }^{[53]}$ Dies führte sogar dazu, dass die Firma sämtliche Aktivitäten stoppen musste. Solche Preisdifferenzen sind für Anwendungen in der Katalyse für großtechnische Produkte oder Spezialitäten weniger kritisch, da die Metallverluste deutlich geringer sind als im Falle der Abgaskatalysatoren. ${ }^{[54]}$

Im Falle von Goldheterogenkatalysatoren wird nach Ende der Katalysatorlebenszeit der gebrauchte Katalysator ausgebaut und wieder aufgearbeitet, um das wertvolle Gold zurückzugewinnen, so wie dies schon heute mit anderen Edelmetallkatalysatoren getan wird. ${ }^{[55]}$ Das heißt, dass für Anwendungen in der chemischen Industrie die Verfügbarkeit der Metalle eine größere Rolle spielt als bloß der Preis. Dies erklärt auch, warum für den Katalysator der PVC-Synthese der maximale Goldgehalt bei $0.25 \%$ liegen durfte. Während die Kosten für den Quecksilberkatalysator 1\% der Gesamtproduktionskosten von PVC ausmachten (Umweltschutzkosten nicht eingerechnet), macht der Goldkatalysator mit dem deutlich teureren Metall jetzt 2\% der Gesamtherstellkosten aus, was gerade noch tragbar ist.

Man wird sich also fragen, ob Goldkatalysatoren in der chemische Industrie eine weite Verbreitung finden werden. Werden leicht wiedergewinnbare nanopartikuläre Goldkatalysatoren, analog zu Palladium, Einzug in andere Anwendungsgebiete der chemischen und pharmazeutischen Industrie halten ? ${ }^{[56]}$ Werden ORMOSIL-verkapselte SiliaCat-AuKatalysatoren $^{[57]}$ oder AUROlite $\left(1 \% \mathrm{Au} / \mathrm{TiO}_{2}\right)^{[58]}$ jemals weitverbreitet in kontinuierlichen Mikroreaktoren eingesetzt werden, und werden dadurch drastische Verringerungen der Reaktionszeiten für die selektive Oxidation von Alkoholen in wässriger Lösung ohne Einsatz von Base erreichbar sein? Es bleibt abzuwarten.

Die Verfügbarkeit von Katalysatoren oder von umweltschonenden Verfahren ist zwar eine notwendige, aber keine hinreichende Bedingung für einen kommerziellen Erfolg. Allein die Tatsache, dass ein Katalysator vorhanden ist, bedeutet noch lange nicht, dass er auch technisch eingesetzt werden wird (siehe den Fall mit den $[\mathrm{L}-\mathrm{Au}]^{+}$-Homogenkatalysatoren). Als erstes muss es einen Bedarf an einem verbesserten Katalysator oder Verfahren geben. Anders ausgedrückt: Ein Katalysatorhersteller wird einen Katalysator erst in sein Portfolio aufnehmen, wenn er dafür eine mögliche Anwendung sieht. In solch einem Fall wird der Hersteller einen eigens für diese Anwendung optimierten Katalysator entwickeln (was in der Regel mit einem großen Aufwand verbunden ist), bevor er ihn kommerziell anbietet.

Bis heute haben nur drei Goldkatalysatoren kommerzielle Anwendung gefunden. Andere, die sogar im Pilotmaßstab getestet worden sind, haben es dagegen nicht geschafft, den strengen Maßstäben zu genügen, unter anderem der 1 Gew.-\% AUROlite-Katalysator für die Hydrierung von $\mathrm{CO}_{2} \mathrm{zu}$ Ameisensäure, der an einer zu niedrigen Aktivität gescheitert ist. ${ }^{[59]}$

Es bleibt abzuwarten, ob in Zukunft die Anzahl technisch eingesetzter Goldkatalysatoren steigen wird, nicht nur bei der Herstellung von Grundchemikalien, sondern vielleicht auch auf anderen Gebieten wie der Bioraffinerie oder bei Fein- chemikalien oder Pharmavorprodukten. Suzuki und Mitarbeiter bei Asahi bescheinigen dem $\mathrm{Au}-\mathrm{NiO}_{x}$-Katalysator eine breite Substratpalette und kündigten an, diesen Katalysator auch für andere Oxidationsreaktionen testen zu wollen. ${ }^{[11]}$ Dies sind Entwicklungen, die 1985 noch unvorstellbar waren, als Hutchings vorhersagte, dass den Standardelektrodenpotentialen zufolge Gold oder sein Chlorid das aktivste Metall für die Acetylen-Hydrochlorierung sein sollte. ${ }^{[60]}$

\section{Danksagung}

M.P. und R.C. danken Dr. Francois Béland und Dr. Valerica Pandarus, SiliCycle (Quebec, Kanada), für die langjährige Zusammenarbeit. Wir danken Prof. Valentine P. Ananikov (Moscow University) für hilfreiche Diskussionen und für die Graphik im Inhaltsverzeichnisbild. Wir danken Professor Emeritus Michele Rossi (Universität Mailand), dessen Leidenschaft für die Goldkatalyse uns alle inspiriert hat.

\author{
Zitierweise: Angew. Chem. Int. Ed. 2016, 55, 14210-14217 \\ Angew. Chem. 2016, 128, 14420-14428
}

[1] K. Sennewald, W. Vogt, H. Glaser, Patent DE1244766B, 1967.

[2] G. C. Bond, P. A. Sermon, Gold Bull. 1973, 6, 102-105.

[3] B. Nkosi, N. J. Coville, G. J. Hutchings, J. Chem. Soc. Chem. Commun. 1988, $71-72$

[4] M. Haruta, T. Kobayashi, H. Sano, N. Yamada, Chem. Lett. 1987, $16,405-408$.

[5] J. H. Teles, S. Brode, M. Chabanas, Angew. Chem. Int. Ed. 1998, 37, 1415-1418; Angew. Chem. 1998, 110, 1475-1478.

[6] J. Oliver-Meseguer, J. R. Cabrero-Antonino, I. Domínguez, A. Leyva-Pérez, A. Corma, Science 2012, 338, 1452-1455.

[7] a) A. S. K. Hashmi, G. J. Hutchings, Angew. Chem. Int. Ed. 2006, 45, 7896-7936; Angew. Chem. 2006, 118, 8064-8105; b) A. S. K. Hashmi, Science 2012, 338, 1434.

[8] A. M. Echavarren, A. S. K. Hashmi, F. D. Toste, Adv. Synth. Catal. 2016, 358, 1347.

[9] P. Johnston, N. Carthey, G. J. Hutchings, J. Am. Chem. Soc. 2015, 137, $14548-14557$.

[10] F. Gao, D. W. Goodman, Chem. Soc. Rev. 2012, 41, 8009-8020.

[11] K. Suzuki, T. Yamaguchi, K. Matsushita, C. Iitsuka, J. Miura, T. Akaogi, H. Ishida, ACS Catal. 2013, 3, 1845-1849.

[12] C. W. Corti, R. J. Holliday, D. T. Thompson, Appl. Catal. A 2005 , $291,253-261$

[13] J. H. Teles, Gold Bull. 2008, 41, 282.

[14] X. Liu, L. He, Y.-M. Liu, Y. Cao, Acc. Chem. Res. 2014, 47, 793 804.

[15] Statista, Global mine production of gold from 2005 to 2015 (in metric tons), New York: 2016. See at the URL: http://www. statista.com/statistics/238414/global-gold-production-since2005/.

[16] J. K. Edwards, B. Solsona, N. B. Ntainjua, A. F. Carley, A. A. Herzing, C. J. Kiely, G. J. Hutchings, Science 2009, 323, 1037 1041.

[17] M. D. Hughes, Y.-J. Xu, P. Jenkins, P. McMorn, P. Landon, D. I. Enache, A. F. Carley, G. A. Attard, G. J. Hutchings, F. King, E. H. Stitt, P. Johnston, K. Griffin, C. J. Kiely, Nature 2005, 437, $1132-1135$.

[18] C. D. Pina, E. Falletta, M. Rossi, Chem. Soc. Rev. 2012, 41, 350 369.

[19] K. Suzuki, Petrotech 2014, 37, 642-646.

[20] S. Yamamatsu, Kagaku Kogyo 2012, 86, 17-24. 
[21] T. Hayashi, T. Inagaki, N. Itayama, H. Baba, Catal. Today 2006, 117, 210-213.

[22] K. Suzuki, T. Yamaguchi, H. Goto, S. Yamane, K. Adachi, Development and commercialization of methyl methacrylate production technology by gold-nickel oxide nanoparticle catalysts with a core-shell structure, Proceedings of 63rd R\&D Symposium of Japan Petroleum Institute, 27 July 2014.

[23] A. Extance, New vinyl catalyst will reduce mercury emissions, Chemistry World, 11. Januar 2016, online verfügbar unter http:// www.rsc.org/chemistryworld/2016/01/gold-catalyst-polyvinylchloride-plastic-industry-china-emission, letzter Zugriff am 27. Juni 2016).

[24] a) K. Saito, C. Fujii, H. Ito, JP 52136103, 1977; b) K. Saito, C. Fujii, JP 52136104, 1977.

[25] G. J. Hutchings, R. Joffe, Appl. Catal. 1986, 20, 215-218.

[26] B. Nkosi, N. J. Coville, G. J. Hutchings, Appl. Catal. 1988, 43, $33-$ 39.

[27] B. Nkosi, N. J. Coville, G. J. Hutchings, M. D. Adams, J. Friedl, F. E. Wagner, J. Catal. 1991, 128, 366-377.

[28] N. A. Carthey, P. Johnston, M. L. Smidt, WO2010/055341 A2, 2010.

[29] Jacobs and Johnson-Matthey, Working together on a mercuryfree VCM catalyst, Instrument on Mercury (INC3), Intergovernmental Negotiating Committee, United Nations Environment Programme, Nairobi, 30. Oktober 2011.

[30] M. Conte, A. F. Carley, C. Heirene, D. J. Willock, P. Johnston, A. A. Herzing, C. J. Kiely, G. J. Hutchings, J. Catal. 2007, 250, $231-239$.

[31] Cardiff University, Gold Catalysts for Vinyl Chloride Manufacture, Research Excellence Framework 2014, REF3b.

[32] P. Bishop, N. A. Carthey, WO 2013/008004, 2013

[33] „Applications and Future Trends in Gold Catalysis“: T. Keel, J. McPherson in Environmental Catalysis Over Gold-Based Materials (Hrsg.: G. Avgouropoulos, T. Tabakova), RSC, Cambridge, 2013, Kap. 7.

[34] S. J. Roberts, G. H. Steinbach, D. Padayachee, J. S. McPherson, G. Pattrick, E. Van Der Lingen, WO 2005/115612, 2005.

[35] L. Croll, B. Billingsley, L. Brey, D. Fansler, P. Martinson, Design and Evaluation of Escape and CBRN Respirator Cartridges Using Nano Gold Carbon Monoxide Oxidation Catalysts, 10th International Symposium on Protection against Chemical and Biological Warfare Agents, Stockholm, 8. -11. Juni 2010.

[36] Eine vertiefte Diskussion findet sich bei: U. Reichert, Implementing the Guideline on the Specification Limits for Residues of Metal Catalysts or Metal Reagents (EMEA/CHMP/SWP/ 4446/2000), Rheinische Friedrich-Wilhelms-Universität Bonn, 2009.

[37] Gold Catalysis: An Homogeneous Approach (Hrsg.: F. D. Toste, V. Michelet), Imperial College Press, London, 2014.

[38] M. Schulz, J. H. Teles, Patent WO 97/21648, 1997.

[39] Y. Fukuda, K. Utimoto, J. Org. Chem. 1991, 56, 3729-3731.
[40] K. Breuer, J. H. Teles, D. Demuth, H. Hibst, A. Schäfer, S. Brode, H. Domgörgen, Angew. Chem. Int. Ed. 1999, 38, $1401-$ 1405; Angew. Chem. 1999, 111, 1497-1502.

[41] A. S. Kashin, V. P. Ananikov, J. Org. Chem. 2013, 78, 1111711125.

[42] E. Gross, J. H.-C. Liu, F. D. Toste, G. A. Somorjai, Nat. Chem. 2012, 4, $947-952$

[43] M. C. B. Jaimes, C. R. N. Böhling, J. M. Serrano-Becerra, S. K. Hashmi, Angew. Chem. Int. Ed. 2013, 52, 7963-7966; Angew. Chem. 2013, 125, 8121-8124.

[44] M. C. B. Jaimes, F. Rominger, M. M. Pereira, R. M. B. Carrilho, S. A. C. Carabineiro, S. K. Hashmi, Chem. Commun. 2014, 50, $4937-4940$

[45] A. Y. Klyushin, M. T. Greiner, X. Huang, T. Lunkenbein, X. Li, O. Timpe, M. Friedrich, M. Hävecker, A. Knop-Gericke, R. Schlögl, ACS Catal. 2016, 6, $3372-3380$.

[46] P. A. Sermon, Gold Bull. 1976, 9, 129-131.

[47] Prof. G. Hutchings, zitiert in: „A new Gold Rush“, 21. Juli 2015. http://www.cardiff.ac.uk/news/view/124836-a-new-gold-rush.

[48] China Council for International Cooperation on Environment and Development, Special Policy Study on Mercury Management in China, Beijing: 2011. URL: http://www.cciced.net/ encciced/policyresearch/report/201205/ P020120529368288424164.pdf.

[49] URL: http://www.indexmundi.com/commodities/?commodity = gold\&months $=180$ (zuletzt aufgerufen am 12. Mai 2016)

[50] Siehe die Charts unter der URL: www.nasdaq.com/markets/ palladium.aspx (zuletzt aufgerufen am 12. Mai 2016).

[51] C. W. Corti, R. J. Holliday, D. T. Thompson, Gold Bull. 2002, 35, $111-117$

[52] „Gold“: R. C. Spurga in Commodity Fundamentals: How to Trade the Precious Metals, Energy, Grain, and Tropical Commodity Markets, Wiley, Hoboken, 2012.

[53] R. Kozarsky, Innovation 2012, 10, 21-24. URL: http://www. innovation-america.org/how-do-you-move-nano-market.

[54] K. Boch, M. Schuster, G. Risse, M. Schwarzer, Anal. Chim. Acta 2002, 459, 257-265.

[55] P. Grumett, Platinum Met. Rev. 2003, 47, 163-166.

[56] T. Mallat, A. Baiker, Annu. Rev. Chem. Biomol. Eng. 2012, 3, $11-28$.

[57] R. Ciriminna, A. Fidalgo, V. Pandarus, F. Béland, L. M. Ilharco, M. Pagliaro, Chem CatChem 2015, 7, 254-260.

[58] J. Ni, W.-J. Yu, L. He, H. Sun, Y. Cao, H.-Y. He, K. N. Fan, Green Chem. 2009, 11, 756-759.

[59] D. Preti, C. Resta, S. Squarcialupi, G. Fachinetti, Angew. Chem. Int. Ed. 2011, 50, 12551-12554; Angew. Chem. 2011, 123, 12759 12762.

[60] G. J. Hutchings, J. Catal. 1985, 96, 292-295.

Eingegangen am 12. Mai 2016

Online veröffentlicht am 14. September 2016 